\title{
Review
}

\section{Cleidocranial dysplasia and RUNX2-clinical phenotype-genotype correlation}

Jaruga A., Hordyjewska E., Kandzierski G., Tylzanowski P. Cleidocranial dysplasia and RUNX2-clinical phenotype-genotype correlation. Clin Genet 2016. (C) John Wiley \& Sons A/S. Published by John Wiley \& Sons Ltd, 2016

Runt-related transcription factor $2(R U N X 2 / C b f a 1)$ is the main regulatory gene controlling skeletal development and morphogenesis in vertebrates. It is located on chromosome 6p21 and has two functional isoforms (type I and type II) under control of two alternate promoters (P1 and P2). Mutations within RUNX2 are linked to Cleidocranial dysplasia syndrome (CCD) in humans. CCD is an autosomal skeletal disorder characterized by several features such as delayed closure of fontanels, dental abnormalities and hypoplastic clavicles. Here, we summarize recent knowledge about $R U N X 2$ function, mutations and their phenotypic consequences in patients.

\section{Conflict of interest}

All authors declare no conflict of interest.

\section{A. Jaruga $a^{a, b}, E$. Hordyjewska ${ }^{a, b}$, G. Kandzierskic and P. Tylzanowski ${ }^{\text {a,d }}$}

aDepartment of Biochemistry and Molecular Biology, Medical University, Lublin, Poland, bPostgraduate School of Molecular Medicine, Warsaw, Poland,

${ }^{\mathrm{C}}$ Children Orthopaedic and Rehabilitation Department, Medical University of Lublin, Lublin, Poland, and 'Laboratory for Developmental and Stem Cell Biology, Department of Development and Regeneration, Skeletal Biology and Engineering Research Centre, University of Leuven, Leuven, Belgium

Key words: CBFA1 - Cleidocranial dysplasia - genetics - RUNX2 skeletal disorders

\author{
Corresponding author: Przemko \\ Tylzanowski, Laboratory for \\ Developmental and Stem Cell Biology, \\ Department of Development and \\ Regeneration, Skeletal Biology and \\ Engineering Research Centre, \\ University of Leuven, Herestraat 49, \\ Leuven, Belgium. \\ Tel: +32 163798 64; \\ fax: +32163462 00; \\ e-mail: przemko@med.kuleuven.be
}

Received 4 February 2016, revised and accepted for publication 28 May 2016
RUNX2 (CBFA1/AML3/PEBP2 $\alpha \mathrm{A}$ ) is one of three members of RUNX family of Runt-related transcription factors (RUNX1-3). RUNX1 participates in hematopoietic stem cell differentiation (1) and mutations in this gene result in myeloid leukemia (2). RUNX2 is necessary for osteoblast differentiation, skeletal morphogenesis (3, 4 ), and mutations in this gene lead to Cleidocranial dysplasia (CCD) in humans (OMIM \#119600). RUNX3 acts as a tumor suppressor gene and was found to be linked to gastric cancer (5). This review will summarize the recent progress in studies of various mutations in RUNX2 and their phenotypic consequences in CCD.

\section{RUNX2 structure}

The human and murine $R U N X 2$ genes are localized on chromosome 6p21 (6) and 17 (7), respectively. Human
RUNX2 gene comprises a region of $223 \mathrm{~kb}$ in size (Chr6:45328317-45551082) (6) and consists of eight exons. Nevertheless, several authors have numbered exons differently depending on the use of alternate promoters and/or alternative splicing yielding 12 transcript variants encoding protein isoforms of RUNX2. For instance, Terry et al. (8) identified two alternate promotors and reported differential utilization of $3^{\prime}$ terminal exons in mouse and human. They also distinguished nine alternatively spliced exons among which exist three variants of exon $5(5,5.1,5.2)$ and two alternatives of exon 6 $(6,6.1)$. Moreover, Makita et al. (9) distinguished three alternatively spliced isoforms that arises from omission of exon 5 and/or exon 7 (figures $(8,9)$ ).

RUNX2 presents two in-frame ATG codons, both of which can serve as potential translation start sites. 


\section{Jaruga et al.}

Two promoters drive expression of both isoforms with different spatiotemporal pattern which suggests specialized functions of each (10). The gene expression from the proximal promoter $(\mathrm{P} 2)$ generates type I RUNX2 mRNA differing at the $5^{\prime}$ end from the type II RUNX2 mRNA being under control of $\mathrm{P} 1$ distal promoter. $\mathrm{P} 1$ promoter is termed the 'bone-related' because of driving expression of the isoform widely associated with bones $(11-16)$.

The translation start codon for the type I RUNX2 is situated within exon 2 and encodes 507-amino acid protein starting with the MRIPV N-terminal sequence (MRIPV isoform; UniProt: Q13950-2) (17). The alternative start codon of type II localizes within exon 1, thus protein begins with the MASNS sequence and is 521-amino acid long (MASNS isoform; UniProt: Q13950-1) (18) (Fig. 1).

In spite of abundance of information, the consistent exon numbering has not been established. Specifically, the exon $1(9,12,14,19,20)$ is termed by some authors 'exon 0' (21-24). Nonetheless, MASNS isoform is indicated as 'canonical' for osteogenesis (13). More importantly, recently NCBI annotation team removed the 507-aa MRIPV annotation (isoform c; NM_004348.3) due to insufficient support for existence of that isoform. Thus, two main remaining isoforms are 521-aa MASNS (isoform 1 or a; NM_001024630.3; NP_001019801.3) and 485-aa MRIPV without seventh exon (isoform 4 or d; NM_001278478.1; NP_001265407) (Fig. 1). Furthermore, NCBI and ENSEMBL nomenclature differs in transcript lengths and there is no clear distinction between type I (MRIPV) and type II (MASNS) RUNX2.

RUNX2 is a multidomain protein which in this review will be described in $\mathrm{N}$ to $\mathrm{C}$ terminal order. The deletion analysis identified the first 19 amino acid fragment as the first activation domain. Next, QA domain composed of glutamine-alanine repeats $(23 \mathrm{Q}$ and $17 \mathrm{~A}$ repeats) is located within N-terminus with a transactivation activity as well. Additionally, it has been shown that this domain can prevent heterodimerization of type II RUNX2 with CBF $\beta$ (core-binding factor subunit beta) (25). This transcriptional partner of RUNX proteins improves its DNA-binding affinity by promoting structural changes that reveal RUNX2-DNA interaction surface (26). CBF $\beta$ also protects and stabilizes RUNX proteins against proteolytic degradation $(27,28)$. CBF $\beta$ does not bind directly to DNA but heterodimerizes with Runt homologous domain (RHD). All members of RUNX family (RUNX1-3) are characterized by the presence of that highly conserved motif, homologous to pair-rule gene runt involved in body segmentation of Drosophila melanogaster (6). RHD consists of 128 amino acids and is responsible for DNA binding (17) to a DNA motif TGXGGTX ( $\mathrm{X}$ is pyrimidine) (29). A 9-amino acid sequence (PRRHRQKLD) following RHD acts as nuclear localization signal (NLS). This domain is related to the NLS of c-Myc, and is conserved within all RUNX proteins (25).

The NLS domain is followed by the proline/serine/threonine rich region (PST) necessary for transcriptional activation of target genes (third transactivation domain) and containing the phosphorylation sites recognized by Akt kinase $(30,31)$. Also ERK/MAPK and PKA kinases phosphorylate RUNX2 protein but in different regions [for review (32)]. Next domain is the nuclear matrix targeting sequence (NMTS). This 38 amino acids motif determines RUNX2 subnuclear localization and displays binding affinity to other proteins like histone deacetylase $(30,33)$. At the very C-terminus

(a)

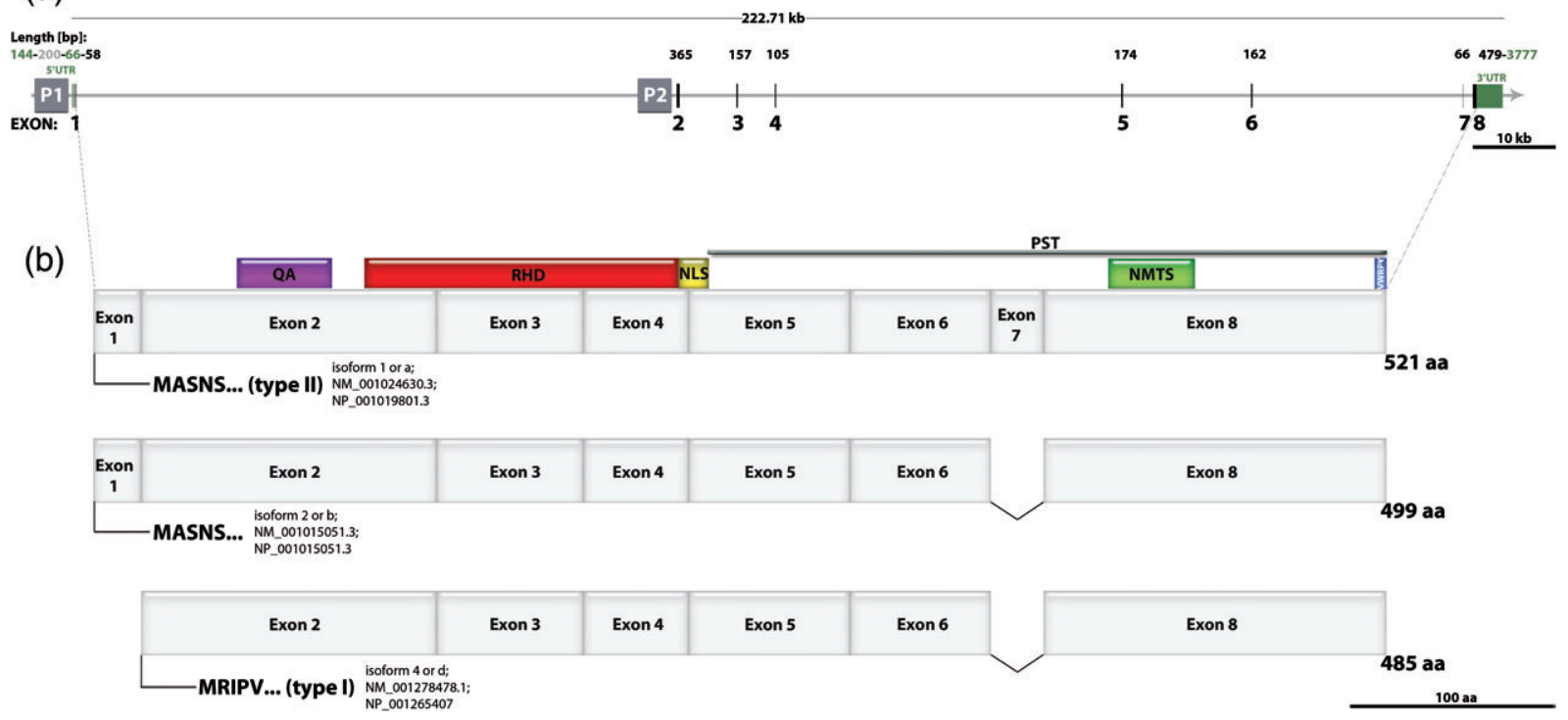

Fig. 1. Schematic representation of RUNX2. (a): RUNX2 gene spans $222.71 \mathrm{~kb}$ and is under the control of two promoters: P1 and P2. Coding sequence are marked as black boxes and length of coding sequences is in block; green boxes indicate $5^{\prime}$ UTR and $3^{\prime}$ UTR, and UTR length is in green; grey line shows introns. (b) Representation of two types of RUNX2: type I and type II starting with MRIPV or MASNS, respectively. QA, polyglutamine and polyalanine domain, RHD, runt homologous domain; NLS, nuclear localization signal; PST, proline/serine/threonine rich region; NMTS, nuclear matrix targeting signal; VWRPY, carboxyterminal pentapetide. 
is present the VWRPY pentapeptide sequence which is recognized as a repression domain. This motif is also conserved among all runt proteins and interacts with transcriptional corepressor TLE2 (transducing-like enhancer of split 2) $(25,34)$ (Fig. 1).

\section{Biological function of RUNX2}

Bones are formed through one of two ossification processes: (i) intramembranous based on direct conversion and differentiation of mesenchymal progenitors into osteoblasts occurring in parts of the craniofacial skeleton as well as the clavicle (35) or (ii) endochondral ossification. In this process, mesenchymal cells differentiate into chondrocytes, which undergo hypertrophy leading among others to the secretion of vascular endothelial growth factor (VEGF) that attracts blood vessels, and permits the formation of bone marrow cavity concomitant with osteoblast differentiation.

RUNX2 is essential for differentiation of osteoblast during both intramembranous and endochondral ossification. Hypertrophy of chondrocytes is positively regulated by RUNX2 which activates the expression of type X collagen (36). Other factors such as SOX9, IHH (Indian hedgehog), PTHRP (parathyroid hormone-related protein) and BMPs (bone morphogenetic proteins) are also responsible for tight regulation of endochondral bone formation (37-40). The initial osteoblast differentiation is triggered through IHH induced by RUNX2 (41-43). RUNX2 activates the IHH promoter and thus stimulates IHH expression in prehypertrophic chondrocytes, which regulates chondrocyte proliferation and maturation through IHH/PTHRP loop (Fig. 2) (44, 45). Additionally, IHH induces expression of RUNX2 in neighboring perichondrium but full commitment to osteoblasts requires Osterix (Osx) transcription factor for both types of ossification (46). Also RUNX2, as a transcription factor involved in bone formation, upregulates the expression of bone matrix genes including collagen I, osteocalcin, bone sialoprotein and osteopontin.

Normal bone development requires precise spatiotemporal regulation of RUNX2 expression and several factors have been associated with this process, and it has been a subject of a recent review (47). Some factors such as Msh homeobox 2 (Msx2), twist or promyelocytic leukemia zinc-finger protein (PLZF), Osx participate in the induction of RUNX2 expression. Others like sex-determining region Y-box 9 (Sox9) acts as a repressor due to interaction with runt domain. Additionally, STAT family transcription factor (Stat1) inhibits nuclear localization of RUNX2. It is worth mentioning that extending recent knowledge about complex regulation of bone formation by transcription factors is essential for further studies in field of TF-targeted gene therapy (48).

RUNX2 type I and type II regulate expression of bone-related genes, however it has been suggested that they might have different functions in skeletogenesis (10). Type I, is expressed in T cells, osteoblasts and chondrocytes (49) and contributes to the intramembranous bone development (50). The expression of Type II increases during osteoblast differentiation $(14,51)$, and it
Cleidocranial dysplasia and RUNX2

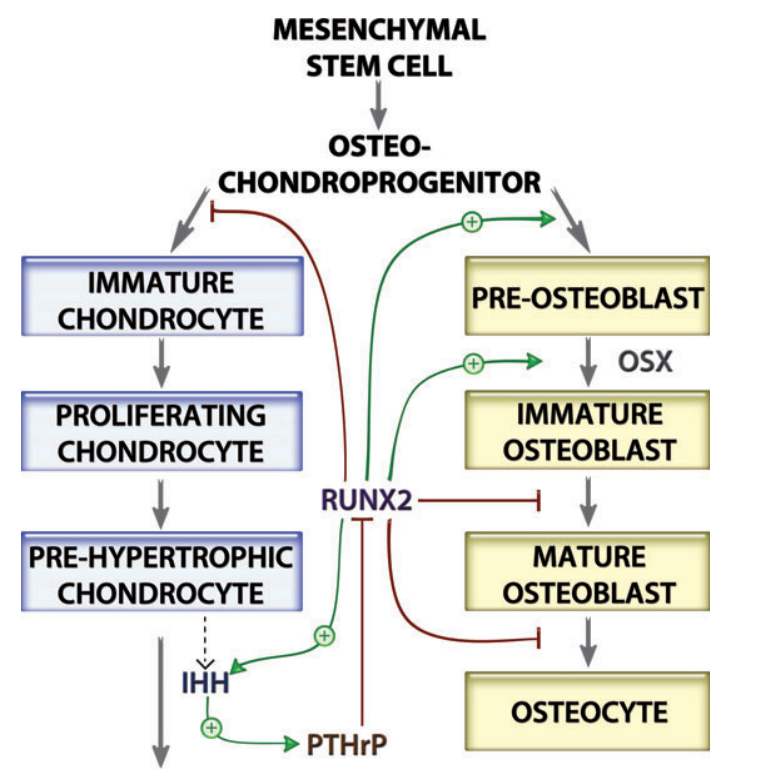

HYPERTROPHIC

CHONDROCYTE

Fig. 2. Regulation of osteoblast and chondrocyte maturation by RUNX2. IHH, Indian hedgehog; PTHrP, parathyroid hormone-related protein; OSX, osterix; green lines indicate induction, red, inhibition and dashed line shows the expression of IHH.

has been shown to be responsible for endochondral bone formation (10). Furthermore, it was reported that these isoforms vary in their dependence on the $\mathrm{Cbf} \beta$ cofactor (52). Moreover, RUNX2 is also responsible for regulating expression of genes that, when deregulated, cause craniosynostosis like NEL-like 1 (NELL1). It also plays a role in hormonal regulation of bone formation by taking part in glucocorticoid signaling pathway necessary for normal osteogenesis (48).

Besides its osteogenic function, RUNX2 has also been linked to breast and prostate cancer and specifically with more aggressive phenotype of these diseases (31, 53-55). Additionally, high expression of RUNX2 is observed in cancers that have high tendency to metastasize to skeleton (56).

\section{Animal models to study Runx2 function}

Targeted inactivation of the gene in mouse results in the phenotype similar to CCD in humans. Specifically, mice were displaying skeletal, CCD-like, abnormalities and had decreased body weight. In heterozygous mice $\left(R U N X 2^{+/-}\right)$formation of the tooth primodorium was delayed but otherwise normal. Since mice present only one dentition, development of supernumerary teeth does not occur like it does in humans with CCD (18). Homozygous (RUNX2-l-) mice died soon after birth, and during postmortal examination, failure in bone ossification was detected (48).

Number of experiments have been performed to establish the role of RUNX2 mutations in CCD pathology. Lou et al. (57) generated a hypomorphic RUNX2 mutant 


\section{Jaruga et al.}

allele $\left(R U N X 2^{\text {neo }}\right)$ where only part of the transcript was processed to full length. This model allowed them to determine the minimal expression level of RUNX2 required for normal skeletal development. Specifically, PGK (cGMP-dependent protein kinase)-driven neomycine resistance cassette (PGKneo) was inserted into intron 7 interfering with splicing and reducing the expression of wild-type mRNA. The insertion of PGKneo into exon 7 resulted in alternative splicing between exon 7 of RUNX2 and neo cassette. This led to expression of mutant protein lacking $\mathrm{C}$-terminus (thus still including NMTS and a repression domain) (57). It is not excluded that observed phenotype can be a dominant negative activity resulting in the production of partial protein. Nonetheless, their findings demonstrate that less than $70 \%$ of wild-type RUNX2 level resulted in CCD phenotype, whereas mice expressing RUNX2 at higher level showed no skeletal abnormalities. Interestingly, it was also reported that RUNX2 overexpression can also cause dysplasia. Experiment resulted in mice suffering from osteopenia along with bone fractures (58).

Bone unrelated function of RUNX2 has been described in zebrafish (Danio rerio) where the gene is an essential determinant for ventral zygotic genes. Importantly, two additional orthologues of mammalian gene called $R U N X 2 a$ and $R U N X 2 b$ sharing sequence and structure homology have been identified in zebrafish but with divergent expression patterns explaining the retention of duplicate genes through evolution (59). Further studies in zebrafish revealed that RUNX2b appears to be the only known regulator of Vent family of transcription factors (60). Members of this family, VOX, VENT and VED, play a role as repressors in dorsoventral axis establishment (61). Depletion of RUNX2b results in loss of expression of VOX, VENT and VED and consequently in embryo dorsalization. Silencing experiments showed that RUNX2b2 regulation of VENT transcription factors is direct, by interacting with promoter. Additionally, rescue experiments in zebrafish using murine RUNX2 type 2 shown that it can substitute for zebrafish orthologues, suggesting that RUNX2 role in axis formation could be evolutionarily conserved (60).

Recently, it has been shown that Runx 2 regulates fate of epithelial cells of mammary glands during breast cancer development. Deletion of Runx2 during pregnancy in mice decreased differentiation by disrupting population of alveolar progenitor cells while exogenous overexpression of Runx2 in mammary epithelial cells impaired milk production. These findings suggest that decreased amount of endogenous Runx2 is required for full differentiation. Additionally, it was observed that overexpression of RUNX2 led to transition changes in normal mammary epithelial cells while its deletion in developing breast cancer inhibited cancerous cellular phenotype (62).

\section{Runx2 in CCD}

CCD was initially described as cleidocranial dysostosis since it was believed to affect only membranous-derived bones. Further clinical studies have shown that the disorder affected skeleton in general, not only skull and clavicles, so it should be considered as dysplasia rather than dysostosis (63). Spectrum of phenotypes is wide, ranging from mildly affected patients with dental and clavicles anomalies only to individuals with severe defects in skeletal development. Intriguingly, these phenotypic variations occur even within one family (64). The most common features of CCD are delayed closure of fontanels and cranial sutures, brachycephaly, depressed nasal bridge, supernumerary teeth, delayed eruption of permanent teeth, Wormian bones and hypoplasia of clavicles (65). Since it was suggested that RUNX2 regulates not only skeleton development but also the expression of mesenchymal tissue, controlling differentiation o dental epithelium, it could partially explain dental abnormalities. Mutations associated with severe dental abnormalities (supernumerary teeth, eruption failure) affect Runt-domain. In contrast, mild dental problems are correlated with mutations outside the Runt-domain (66-68).

\section{Mutations in human RUNX2}

Majority of RUNX2 mutations in individuals suffering from classic CCD occur in the runt domain and the most common DNA disruptions are missense mutations that prevent RUNX2 binding to DNA or nonsense mutations that result in biosynthesis of truncated protein. While missense mutations are uniquely found within runt-domain region, nonsense and frame-shift types were described throughout the gene $(22,63,66$, 69-80) (Fig. 3, Table 1).

A screening analysis within a group of Japanese patients was carried out to investigate the link between a mutation and its phenotypic manifestation (67). It described 15 heterozygous mutations among 24 families and 1 polymorphism. Most of discovered mutations were missense and all of them located within runt-domain which confirmed results reported previously (81). Besides six missense, seven nonsense and frame-shift mutations, two exon-skipping mutations between third exon and intron were also identified. One of them, IVS3+3delAAGT, occurred within splice-donor signal leading to in-frame skipping of the complete third exon. This resulted in decreased functionality of donor site where GT motif was followed by segment rich in pyrimidine (AAGTACTC) (67). Second exon-skipping mutation, cIVS3+2T $>\mathrm{C}$, was present at the same donor site. It is worth mentioning that different study also described skipping of exon 5 although in this case runt domain and NLS remained intact (81). Extensive genetic analysis of patient groups with CCD led to discovery of several polymorphisms. D170, synonymous mutation, caused by c510T $>\mathrm{C}$ was identified within second exon on one allele in one patient. Mother of the patients was a homozygous carrier of this mutation as well (67). In the second case, polymorphism - A80 $(\mathrm{c} 240 \mathrm{~A}>\mathrm{G})$ - was found on one allele of patient and the mother. The causative silent transition was located within Q/A region (65). Besides the missense mutations (Table 1), several other mutations have been reported such as a mutation in runt-domain, T200A that does 
Cleidocranial dysplasia and RUNX2

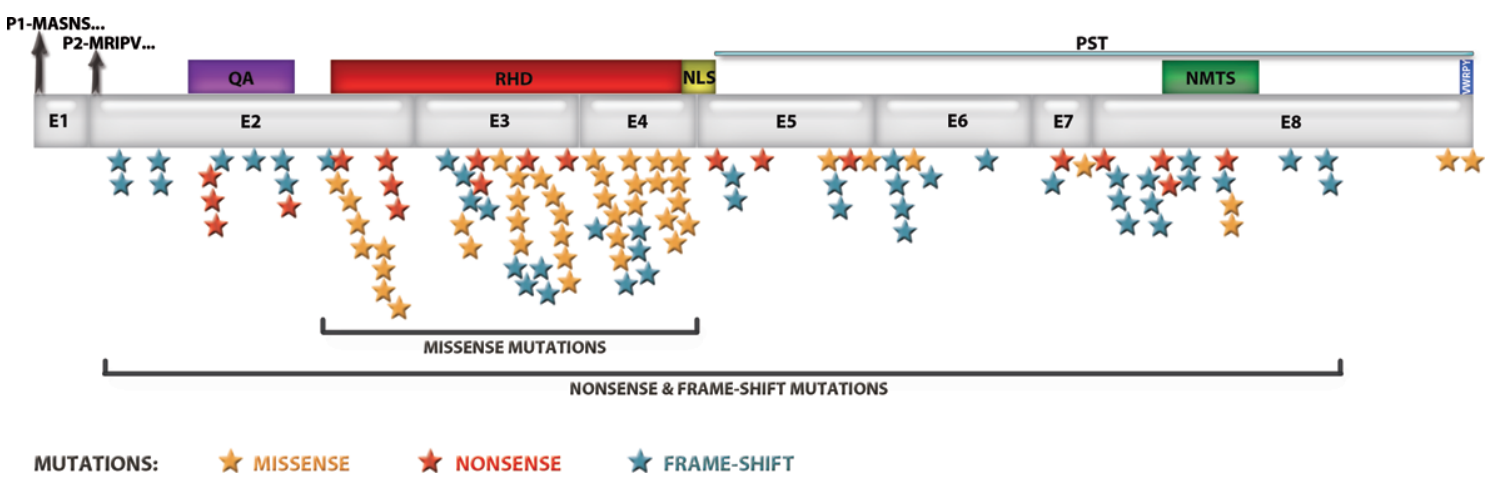

Fig. 3. Schematic representation of mutation types within RUNX2 gene. QA, polyglutamine and polyalanine domain; RHD, runt homologous domain; NLS, nuclear localization signal; PST, proline/serine/threonine rich region; NMTS, nuclear matrix targeting signal VWRPY-carboxyterminal pentapetide.

not affect binding to DNA and is correlated with mild CCD phenotype manifested only by delayed eruption of permanent teeth (81). Electrophoretic mobility shift assay (EMSA) of wild-type and mutant protein revealed that T200A mutation did not affect neither the binding to OSE2 nor transactivation activity. In vivo effect of T200A mutation remains thus unknown. One could speculate that the mutation may be hypomorphic but in vitro studies are not sufficiently sensitive. Alternatively, the mutation may have neomorphic nature and affect different functions of runt domain (81).

Besides point mutations, intragenic microdeletions have been also reported for RUNX2 and linked to CCD. A family has been identified having intragenic deletion covering region between exons 2 and 6, including runt domain and NLS. Mutation was detected within three generations of this family and all patients presented short stature and small hands $(82,83)$. Another case of microdeletion involved not only RUNX2 but also other gene, VEGF, located 4.6 Mb away. Phenotype reflected the defects for both genes: characteristic features of CCD and degenerated pattering of pharyngeal arch-derived arteries, characteristic for VEGF, were observed (84).

Besides microdeletion, the genomic region containing RUNX2 has also been microduplicated. Recently, large duplication downstream of RUNX2 has been discovered in CCD patients who suggested that regulatory sequences of RUNX2 may be disrupted in this case. Family did not participate in further analysis, so segregation analysis and additional gene diagnostics of a possible pathogenic effect of the duplicated region were not carried out (85).

Moreover, a case of family where three children, one of them a half-brother, presented all features of classic CCD phenotype while the mother examination showed no abnormalities (86). Sequencing analysis revealed 864insC mutation within exon 6 in affected family members and a single nucleotide polymorphism IVS6+46G $>A$ in the same exon in the mother. High resolution melting analysis was performed in order to test for maternal mosaicism. Results, showing different melting profiles in each patient, pointed to a possibility of additional sequence variation in mother. Further, subcloning and resequencing identified 864insC mutation in several clones isolated from blood or buccal swab samples. It was found in cis to IVS6+46G allele therefore confirming low-level mosaicism in mother (86).

Recently, a novel duplication 181_189dupGCGGGC GGCT was reported resulting in expansion of polyalanine residues within Q/A domain and, in consequence, in CCD phenotype. Further experiments confirmed that increasing number of alanine residues has a significant impact on RUNX2 transcriptional activity. Expansion by 3 Ala did not impair the normal activity of RUNX2 while 10 Ala expansion significantly decreased it. The Ala expansion caused rapid intracellular aggregation of RUNX2 resulting in the protein exclusion from the nuclei (87). Recently, the plausible connection between the number of alanine repeats within QA domain and the absence of clavicles in patients with CCD has been revealed. The analysis of polyalaninie tracts in warm blooded mammals (including human) revealed that species having 17 or 18 alanine tracts have clavicles, while the absence of repeats or its increased number is associated with the reduction or lack of clavicles (88). Polyalanine repeats however are not the only ones linked with CCD phenotype since a case of polyglutamine tract insertion was reported (213_214insCAGCAGCAGCAG), also in Q/A domain, resulting in reduced transactivation of RUNX2 (89).

\section{Genotype-phenotype correlation}

It has been notoriously difficult to establish direct genotype-phenotype correlation for RUNX2 because of very variable phenotypic penetrance of the mutations (4). Most mutations occurring within runt-domain result in a classical CCD phenotype. There are however some intriguing exceptions like heterozygous T200A mutation. It was found in father who suffered only from dental abnormalities, but his two children showed all the features of CCD (81). Another example of an unusual genotype-phenotype correlation is an insertion mutation localized at the beginning of the transcript, between two translational start sites, 90insC. It was described to be associated with mild CCD phenotype and abnormal dentition (81). Another study investigated phenotype-genotype correlation in a large group of patients. Patients from one group had mutations within 


\section{Jaruga et al.}

Table 1. Precise location of mutations within RUNX2 gene ${ }^{\mathrm{a}}$

\begin{tabular}{|c|c|c|c|}
\hline \multirow[b]{2}{*}{ Exon number } & \multicolumn{3}{|c|}{ Mutation type } \\
\hline & Nonsense & Missense & Frame-shift \\
\hline \multicolumn{4}{|l|}{$1(1-19$ aa) } \\
\hline \multirow[t]{10}{*}{$2(20-141$ aa) } & c.190 C>T [Q64X] & c.338T>G [L113R] & c.90_91insC \\
\hline & c.193 C>T [Q65X]] & c.354 C>T [S118R] & c.92_93insCGGT \\
\hline & c.196 C>T [Q66X] & c.362T>G [F121C] & c. 134 _135insGTCC \\
\hline & c. $273 T>A[L 93 X]$ & c.366_367delGTinsTC & c.136delC \\
\hline & c. $276 \_277$ ins 17 & [C123R] & c.207_208ins13 \\
\hline & {$[\mathrm{L93X}]$} & c.391 C>G [R131G] & c.230-276del \\
\hline & c.334G $>\mathrm{T}[\mathrm{E} 112 \mathrm{X}]$ & c.391 C>T [ R131C] & c. 274 delC \\
\hline & c. 383 C>A [S128X] & c.391 C>A [R131S] & c.282delG \\
\hline & c.389G >A [W130X] & c.407T>C [L136P] & c.329delC \\
\hline & c.396 C>A [C132X] & & \\
\hline \multirow[t]{13}{*}{3 (142-194 aa) } & c.481-482delGA & c.467T>A [V156D] & c.453delT \\
\hline & [D161X] & c. $475 \mathrm{G}>\mathrm{C}$ [G159R] & c.469insA \\
\hline & c.484G>T [E162X] & c.506G>C [R169P] & c.476delG \\
\hline & c.535 C>T [R179X] & c.523 A>G [M175V] & c.495delT \\
\hline & c.577 C>T [R193X] & c.524T>A [M175L] & c.522insA \\
\hline & & c.524T>G [M175R] & c.532delC \\
\hline & & c.526 C>T [R176W] & c.549delC \\
\hline & & c.548T>C [F183S] & c.553_554delCT \\
\hline & & c.560T>C [F187S] & \\
\hline & & c.568C >T [R190W] & \\
\hline & & c.569G >A [R190Q] & \\
\hline & & c. $572 \mathrm{G}>\mathrm{A}[\mathrm{S} 191 \mathrm{~N}]$ & \\
\hline & & c.578G >A [R193Q] & \\
\hline \multirow[t]{18}{*}{4 (194-229 aа) } & & c.590T>C [F197S] & c.592delA \\
\hline & & c.599C>T [T200I] & c.624_625insCC \\
\hline & & c. $602 \mathrm{~T}>\mathrm{A}[\mathrm{l} 201 \mathrm{~K}]$ & c.635_638delCCTA \\
\hline & & c.612A>T [K204N] & c.636delC \\
\hline & & c.614C>G [T205R] & c.644delG \\
\hline & & c.617 C>T [T206I] & \\
\hline & & c.627 A>T [Q209H] & \\
\hline & & c. $631 \mathrm{G}>\mathrm{C}[\mathrm{A} 211 \mathrm{P}]$ & \\
\hline & & c. $632 \mathrm{G}>\mathrm{A}[\mathrm{R} 211 \mathrm{Q}]$ & \\
\hline & & c. 635- 638delCCTA [T212I] & \\
\hline & & c.652 A >C [L218Q] & \\
\hline & & c.652 A > G [L218E] & \\
\hline & & c. $662 \mathrm{~T}>\mathrm{G}[\mathrm{V} 221 \mathrm{G}]$ & \\
\hline & & c. $667 \mathrm{G}>\mathrm{A}[\mathrm{G} 223 \mathrm{R}]$ & \\
\hline & & c. $673 \mathrm{C}>\mathrm{T}[\mathrm{R} 225 \mathrm{~W}]$ & \\
\hline & & c. $674 G>A[R 225 Q]$ & \\
\hline & & c. $674 G>T$ [R225L] & \\
\hline & & c.682 A>G [R228G] & \\
\hline \multirow[t]{4}{*}{5 (229-287 aа) } & c.694 C>T [Q232X] & c. $817 \mathrm{G}>\mathrm{A}[\mathrm{D} 273 \mathrm{~N}]$ & c.718_721del \\
\hline & c.793 C>T [Q266X] & c. $859 \mathrm{G}>\mathrm{A}[\mathrm{D} 287 \mathrm{~N}]$ & c.722delT \\
\hline & c.838 C>T [Q280X] & & c.824delG \\
\hline & & & c.831delT \\
\hline \multirow[t]{6}{*}{$6(287-341$ aa) } & & c.896 C>T [P299L] & c.873_874delCA \\
\hline & & & c.879_885del \\
\hline & & & c.884delC \\
\hline & & & c.887delC \\
\hline & & & c.915delC \\
\hline & & & c. $977 \mathrm{delG}$ \\
\hline \multirow{13}{*}{$8(363-521 \mathrm{aa})$} & c. $1056 \mathrm{G}>\mathrm{A}[\mathrm{W} 352 \mathrm{X}]$ & c.1085 C>T [A362V] & c. 1043 C>T (splice region variant) \\
\hline & c. $1096 \mathrm{G}>\mathrm{T}[\mathrm{E} 366 \mathrm{X}]$ & c. 1259 C>A [T420N] & c.1111_1129del19 \\
\hline & c. 1171 C>T [R391X] & C. $1259 \mathrm{C}>\mathrm{T}$ [T420I] & c.1119delC \\
\hline & c.1182T>A [Y394X] & c. $1531 \mathrm{G}>\mathrm{A}[\mathrm{G} 511 \mathrm{~S}]$ & c.1127_1128insT \\
\hline & c. 1254 C>A [Y418X] & $1565 G>C$ [X522S] (stop lost) & c.1153_1154delAG \\
\hline & & & C. 1157 delG \\
\hline & & & C.1169delC \\
\hline & & & c.1205delC \\
\hline & & & c.1205_1206insC \\
\hline & & & c.1250_1251delAC \\
\hline & & & c.1335_1336delCT \\
\hline & & & c.1379_1380insC \\
\hline & & & c.1385insG \\
\hline
\end{tabular}

aThe table contains the data as available in public databases on April 2016. 
Cleidocranial dysplasia and RUNX2

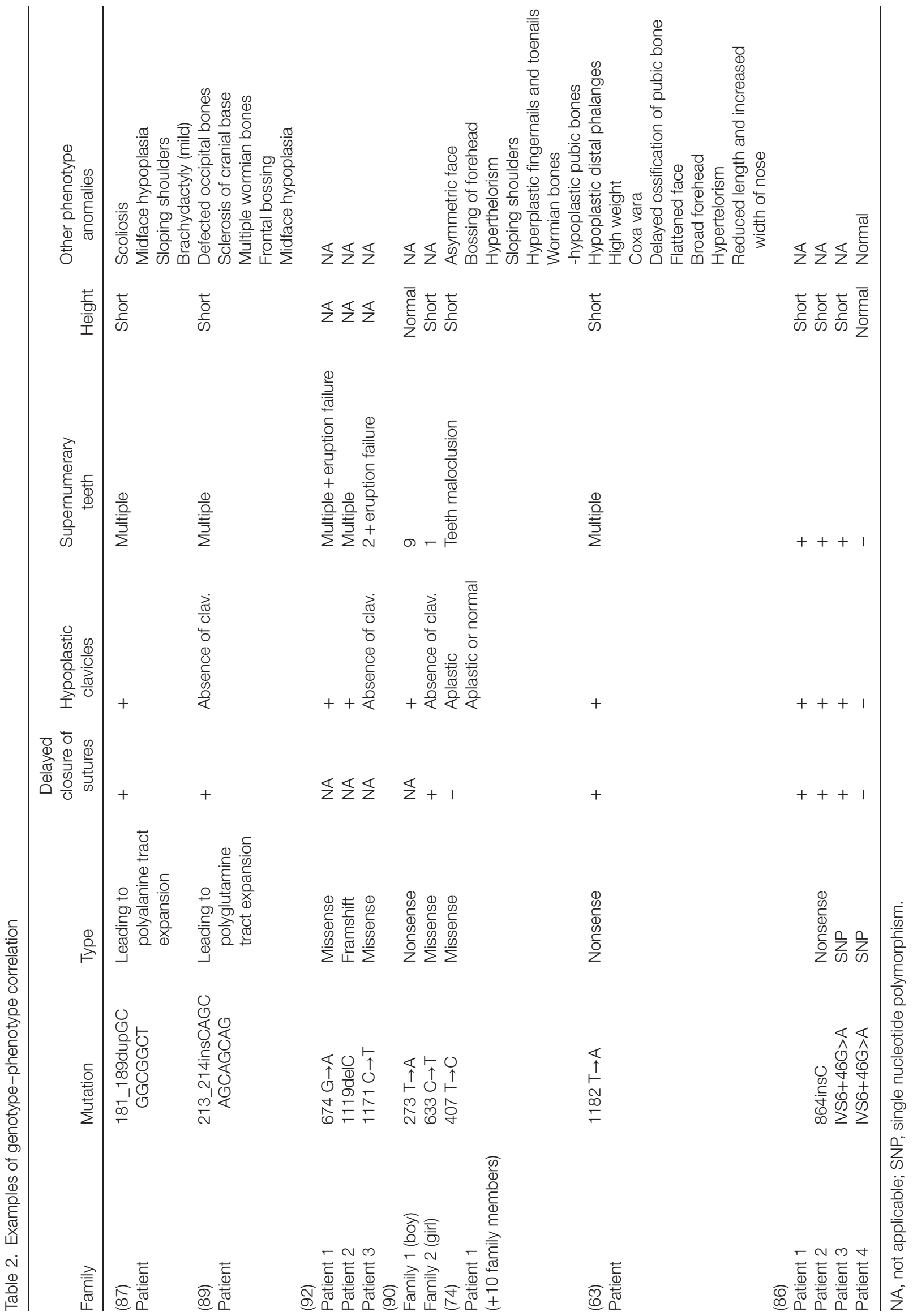




\section{Jaruga et al.}

runt-domain. Individuals from the second group had runt-domain unaffected while did have all the features of CCD. This suggests that phenotype may be caused by mutation in a regulatory region on RUNX2 or in other gene interacting with RUNX2. Besides the range of CCD phenotypes present in these patients, most common difference was the height score, significantly lower in group with impaired runt-domain. Findings suggested that growth of the skeleton deteriorates when one allele activity is lower by around 50\% and it progressively worsens with further loss of gene activity (67).

There is also a weak genotype-phenotype correlation in case of dental aspect of CCD phenotypes, especially with respect to teeth development (90). Among several hypotheses trying to explain phenotype diversity, one suggests that intramembranous bone formation may require more RUNX2 in comparison to endochondral-mediated skeletal bones development (67). Additionally, recent report demonstrates that there are more dental-related phenotypes correlated with CCD describing a sporadic case of 14 supernumerary teeth in 20-year old individual. Patient was found with, previously unreported, fused primary teeth due to c.578G >A (R193Q) mutation (68). There are many similarities between osteo- and odontogenesis. This, together with the role that osteoblasts play in the formation of mandible and maxilla, explains the severity of craniofacial phenotypes in CCD patients (91).

In summary, RUNX2 is a master regulatory gene for skeletal development and morphogenesis. Mutations within the gene or its regulatory regions are commonly found in CCD patients. Further studies are however necessary to establish in more detail the genotype-phenotype correlation (Table 2) $(63,74,86$, $87,89,90,92)$ and to fully understand mechanism of underlying pathogenesis of CCD.

\section{Acknowledgements}

This study was partially funded by $\mathrm{NCN}$ grant number 2013/11/B/NZ4/03660.

\section{References}

1. Okuda T, vanDeursen J, Hiebert SW et al. AML1, the target of multiple chromosomal translocations in human leukemia, is essential for normal fetal liver hematopoiesis. Cell 1996: 84: 321-330.

2. Miyoshi H, Shimizu K. T. K et al. $\mathrm{t}(8 ; 21)$ breakpoints on chromosome 21 in acute myeloid leukemia are clustered within a limited region of a single gene, AML1. Proc Natl Acad Sci USA 1991: 88: 10431-10434.

3. Komori T, Yagi H, Nomura S et al. Targeted disruption of Cbfa1 results in a complete lack of bone formation owing to maturational arrest of osteoblasts. Cell 1997: 89: 755-764.

4. Otto F, Thornell AP, Crompton T et al. Cbfa1, a candidate gene for cleidocranial dysplasia syndrome, is essential for osteoblast differentiation and bone development. Cell 1997: 89: 765-771.

5. Li QL, Ito K, Sakakura C et al. Causal relationship between the loss of RUNX3 expression and gastric cancer. Cell 2002: 109: 113-124.

6. Levanon D, Negreanu V, Bernstein Y et al. AML1, AML2, and AML3, the human members of the runt domain gene-family: cDNA structure, expression, and chromosomal localization. Genomics 1994: 23: 425-432.

7. Mundlos S, Mulliken JB, Abramson DL et al. Genetic mapping of cleidocranial dysplasia and evidence of a microdeletion in one family. Hum Mol Genet 1995: 4: 71-75.
8. Terry A, Kilbey A, Vaillant F et al. Conservation and expression of an alternative 3' exon of Runx2 encoding a novel proline-rich C-terminal domain. Gene 2004: 336: 115-125.

9. Makita N, Suzuki M, Asami S et al. Two of four alternatively spliced isoforms of RUNX2 control osteocalcin gene expression in human osteoblast cells. Gene 2008: 413: 8-17.

10. Xiao ZS, Hjelmeland AB, Quarles LD. Selective deficiency of the "bone-related" Runx2-II unexpectedly preserves osteoblast-mediated skeletogenesis. J Biol Chem 2004: 279: 20307-20313.

11. Stewart M, Terry A, Hu M et al. Proviral insertions induce the expression of bone-specific isoforms of PEBP2 alpha A (CBFA1): evidence for a new myc collaborating oncogene. Proc Natl Acad Sci USA 1997: 94: 8646-8651.

12. Drissi H, Luc Q, Shakoori R et al. Transcriptional autoregulation of the bone related CBFA1/RUNX2 gene. J Cell Physiol 2000: 184: 341-350.

13. Xiao ZS, Liu SG, Hinson TK et al. Characterization of the upstream mouse Cbfa1/Runx2 promoter. J Cell Biochem 2001: 82: 647-659.

14. Banerjee C, Javed A, Choi JY et al. Differential regulation of the two principal Runx2/Cbfa1 n-terminal isoforms in response to bone morphogenetic protein-2 during development of the osteoblast phenotype. Endocrinology 2001: 142: 4026-4039.

15. Tamiya H, Ikeda T, Jeong JH et al. Analysis of the Runx2 promoter in osseous and non-osseous cells and identification of HIF2A as a potent transcription activator. Gene 2008: 416: 53-60.

16. Liu JC, Lengner CJ, Gaur T et al. Runx 2 protein expression utilizes the Runx2 P1 promoter to establish osteoprogenitor cell number for normal bone formation. J Biol Chem 2011: 286: 30057-30070.

17. Ogawa E, Maruyama M, Kagoshima $\mathrm{H}$ et al. PEBP2/PEA2 represents a family of transcription factors homologous to the products of the Drosophila runt gene and the human AMLI gene. Proc Natl Acad Sci USA 1993: 90: 6859-6863.

18. Mundlos S, Otto F, Mundlos C et al. Mutations involving the transcription factor CBFA1 cause cleidocranial dysplasia. Cell 1997: 89: $773-779$.

19. Levanon D, Groner Y. Structure and regulated expression of mammalian RUNX genes. Oncogene 2004: 23: 4211-4219.

20. Cunningham ML, Seto ML, Hing AV et al. Cleidocranial dysplasia with severe parietal bone dysplasia: C-terminal RUNX2 mutations. Birth Defects Res A Clin Mol Teratol 2006: 76: 78-85.

21. Park MH, Shin HI, Choi JY et al. Differential expression patterns of Runx2 isoforms in cranial suture morphogenesis. J Bone Miner Res 2001: 16: 885-892.

22. Otto F, Kanegane H, Mundlos S. Mutations in the RUNX2 gene in patients with cleidocranial dysplasia. Hum Mutat 2002: 19: 209-216.

23. Yoshida $\mathrm{T}$, Kanegane $\mathrm{H}$, Osato $\mathrm{M}$ et al. Functional analysis of RUNX2 mutations in cleidocranial dysplasia: novel insights into genotype-phenotype correlations. Blood Cells Mol Dis 2003: 30: $184-193$.

24. Kim HJ, Nam SH, Kim HJ et al. Four novel RUNX2 mutations including a splice donor site result in the cleidocranial dysplasia phenotype. J Cell Physiol 2006: 207: 114-122.

25. Thirunavukkarasu K, Mahajan M, McLarren KW et al. Two domains unique to osteoblast-specific transcription factor Osf2/Cbfa1 contribute to its transactivation function and its inability to heterodimerize with Cbfb. Mol Cell Biol 1998: 18: 4197-4208.

26. Tahirov TH, Inoue-Bungo $\mathrm{T}$, Morii $\mathrm{H}$ et al. Structural analyses of DNA recognition by the AML1/Runx-1 Runt domain and its allosteric control by CBFbeta. Cell 2001: 104: 755-767.

27. Park J, Gebhardt M, Golovchenko S et al. Dual pathways to endochondral osteoblasts: a novel chondrocyte-derived osteoprogenitor cell identified in hypertrophic cartilage. Biol Open 2015: 4: 608-621.

28. Kagoshima H, Shigesada K, Satake M et al. The Runt domain identifies a new family of heteromeric transcriptional regulators. Trends Genet 1993: 9: $338-341$.

29. Crute BE, Lewis $\mathrm{AF}, \mathrm{Wu} \mathrm{Z}$ et al. Biochemical and biophysical properties of the core-binding factor alpha2 (AML1) DNA-binding domain. J Biol Chem 1996: 271: 26251-26260.

30. Kanno T, Kanno $\mathrm{Y}$, Chen LF et al. Intrinsic transcriptional activation-inhibition domains of the polyomavirus enhancer binding protein $2 /$ core binding factor alpha subunit revealed in the presence of the beta subunit. Mol Cell Biol 1998: 18: 2444-2454.

31. Pande S, Browne G, Padmanabhan S et al. Oncogenic cooperation between PI3K/Akt signaling and transcription factor Runx2 promotes the invasive properties of metastatic breast cancer cells. J Cell Physiol 2013: 228: 1784-1792. 


\section{Cleidocranial dysplasia and RUNX2}

32. Vimalraj S, Arumugam B, Miranda PJ et al. Runx2: structure, function, and phosphorylation in osteoblast differentiation. Int J Biol Macromol 2015: 78: 202-208.

33. Zaidi SK, Javed A, Choi JY et al. A specific targeting signal directs Runx2/Cbfa1 to subnuclear domains and contributes to transactivation of the osteocalcin gene. J Cell Sci 2001: 114: 3093-3102.

34. Levanon D, Goldstein RE, Bernstein Y et al. Transcriptional repression by AML1 and LEF-1 is mediated by the TLE/Groucho corepressors. Proc Natl Acad Sci USA 1998: 95: 11590-11595.

35. Olsen BR, Reginato AM, Wang W. Bone development. Annu Rev Cell Dev Biol 2000: 16: 191-220.

36. Zheng $\mathrm{Q}$, Zhou G, Morello $\mathrm{R}$ et al. Type $\mathrm{X}$ collagen gene regulation by Runx 2 contributes directly to its hypertrophic chondrocyte-specific expression in vivo. J Cell Biol 2003: 162: 833-842.

37. Franceschi RT, Xiao G. Regulation of the osteoblast-specific transcription factor, Runx2: responsiveness to multiple signal transduction pathways. J Cell Biochem 2003: 88: 446-454.

38. Lee MH, Kim YJ, Kim HJ et al. BMP-2-induced Runx2 expression is mediated by Dlx5, and TGF-beta 1 opposes the BMP-2-induced osteoblast differentiation by suppression of Dlx 5 expression. J Biol Chem 2003: 278: 34387-34394.

39. Nishimura R, Hata K, Matsubara T et al. Regulation of bone and cartilage development by network between BMP signalling and transcription factors. J Biochem 2012: 151: 247-254.

40. Lefebvre V, Smits P. Transcriptional control of chondrocyte fate and differentiation. Birth Defects Res C Embryo Today 2005: 75: 200-212.

41. Vortkamp A, Lee K, Lanske B et al. Regulation of rate of cartilage differentiation by Indian hedgehog and PTH-related protein. Science 1996: 273: 613-622.

42. Enomoto H, Enomoto-Iwamoto M, Iwamoto $\mathrm{M}$ et al. Cbfa1 is a positive regulatory factor in chondrocyte maturation. J Biol Chem 2000: 275: 8695-8702

43. St-Jacques B, Hammerschmidt M, McMahon AP. Indian hedgehog signaling regulates proliferation and differentiation of chondrocytes and is essential for bone formation. Genes Dev 1999: 13: 2072-2086.

44. Yoshida CA, Yamamoto H, Fujita T et al. Runx2 and Runx3 are essential for chondrocyte maturation, and Runx2 regulates limb growth through induction of Indian hedgehog. Genes Dev 2004: 18: 952-963.

45. Iwamoto M, Kitagaki J, Tamamura $\mathrm{Y}$ et al. Runx 2 expression and action in chondrocytes are regulated by retinoid signaling and parathyroid hormone-related peptide (PTHrP). Osteoarthritis Cartilage 2003: 11: $6-15$.

46. Nakashima K, Zhou X, Kunkel G et al. The novel zinc finger-containing transcription factor Osterix is required for osteoblast differentiation and bone formation. Cell 2002: 108: 17-29.

47. Bruderer M, Richards RG, Alini M et al. Role and regulation of RUNX2 in osteogenesis. Eur Cell Mater 2014: 28: 269-286.

48. Liu TM, Lee EH. Transcriptional regulatory cascades in Runx2-dependent bone development. Tissue Eng Part B Rev 2013: 19 254-263.

49. Harada H, Tagashira S, Fujiwara M et al. Cbfa1 isoforms exert functional differences in osteoblast differentiation. J Biol Chem 1999: 274 6972-6978.

50. Choi KY, Lee SW, Park MH et al. Spatio-temporal expression patterns of Runx2 isoforms in early skeletogenesis. Exp Mol Med 2002: 34 $426-433$.

51. Stein GS, Lian JB, van Wijnen AJ et al. Runx2 control of organization, assembly and activity of the regulatory machinery for skeletal gene expression. Oncogene 2004: 23: 4315-4329.

52. Kanatani N, Fujita T, Fukuyama R et al. Cbf beta regulates Runx2 function isoform-dependently in postnatal bone development. Dev Biol 2006: 296: 48-61

53. Pratap J, Javed A, Languino LR et al. The Runx 2 osteogenic transcription factor regulates matrix metalloproteinase 9 in bone metastatic cancer cells and controls cell invasion. Mol Cell Biol 2005: 25: 8581-8591.

54. Akech J, Wixted JJ, Bedard K et al. Runx2 association with progression of prostate cancer in patients: mechanisms mediating bone osteolysis and osteoblastic metastatic lesions. Oncogene 2010: 29: 811-821.

55. Tandon M, Chen Z, Pratap J. Runx2 activates PI3K/Akt signaling via mTORC2 regulation in invasive breast cancer cells. Breast Cancer Res 2014: 16: R16.

56. Pratap J, Lian JB, Javed A et al. Regulatory roles of Runx2 in metastatic tumor and cancer cell interactions with bone. Cancer Metastasis Rev 2006: 25: 589-600
57. Lou Y, Javed A, Hussain S et al. A Runx 2 threshold for the cleidocranial dysplasia phenotype. Hum Mol Genet 2009: 18: 556-568.

58. Liu W, Toyosawa S, Furuichi $\mathrm{T}$ et al. Overexpression of Cbfal in osteoblasts inhibits osteoblast maturation and causes osteopenia with multiple fractures. J Cell Biol 2001: 155: 157-166.

59. Flores MV, Tsang VW, Hu W et al. Duplicate zebrafish runx2 orthologues are expressed in developing skeletal elements. Gene Expr Patterns 2004: 4: 573-581.

60. Flores MV, Lam EY, Crosier KE et al. Osteogenic transcription factor Runx2 is a maternal determinant of dorsoventral patterning in zebrafish. Nat Cell Biol 2008: 10: 346-352.

61. Gilardelli CN, Pozzoli O, Sordino P et al. Functional and hierarchical interactions among zebrafish vox/vent homeobox genes. Dev Dyn 2004 230: 494-508.

62. Owens TW, Rogers RL, Best SA et al. Runx2 is a novel regulator of mammary epithelial cell fate in development and breast cancer. Cancer Res 2014: 74: 5277-5286

63. Lo Muzio L, Tete S, Mastrangelo F et al. A novel mutation of gene CBFA1/RUNX2 in cleidocranial dysplasia. Ann Clin Lab Sci 2007: 37 $115-120$.

64. Quack I, Vonderstrass B, Stock M et al. Mutation analysis of core binding factor A1 in patients with cleidocranial dysplasia. Am J Hum Genet 1999 65: $1268-1278$.

65. Zhang C, Zheng S, Wang Y et al. Mutational analysis of RUNX2 gene in Chinese patients with cleidocranial dysplasia. Mutagenesis 2010: 25: 589-594.

66. Bufalino A, Paranaiba LM, Gouvea AF et al. Cleidocranial dysplasia: oral features and genetic analysis of 11 patients. Oral Dis 2012: 18 : 184-190.

67. Yoshida T, Kanegane H, Osato M et al. Functional analysis of RUNX2 mutations in Japanese patients with cleidocranial dysplasia demonstrates novel genotype-phenotype correlations. Am J Hum Genet 2002: 71 : $724-738$.

68. Lu H, Zeng B, Yu D et al. Complex dental anomalies in a belatedly diagnosed cleidocranial dysplasia patient. Imaging Sci Dent 2015: 45: $187-192$.

69. Guo YW, Chiu CY, Liu CL et al. Novel mutation of RUNX2 gene in a patient with cleidocranial dysplasia. Int J Clin Exp Pathol 2015: 8: 1057-1062

70. Fang CY, Xue JJ, Tan L et al. A novel single-base deletion mutation of the RUNX2 gene in a Chinese family with cleidocranial dysplasia. Genet Mol Res 2011: 10: 3539-3544.

71. Xuan D, Li S, Zhang X et al. Mutations in the RUNX2 gene in Chinese patients with cleidocranial dysplasia. Ann Clin Lab Sci 2008: 38: 15-24.

72. Huang $\mathrm{Y}$, Song $\mathrm{Y}$, Zhang $\mathrm{C}$ et al. Novel RUNX2 frameshift mutations in Chinese patients with cleidocranial dysplasia. Eur J Oral Sci 2013: 121: $142-147$.

73. Xuan D, Sun X, Yan Y et al. Effect of cleidocranial dysplasia-related novel mutation of RUNX2 on characteristics of dental pulp cells and tooth development. J Cell Biochem 2010: 111: 1473-1481.

74. Tang S, Xu Q, Xu X et al. A novel RUNX2 missense mutation predicted to disrupt DNA binding causes cleidocranial dysplasia in a large Chinese family with hyperplastic nails. BMC Med Genet 2007: 8: 82.

75. Tessa A, Salvi S, Casali C et al. Six novel mutations of the RUNX2 gene in Italian patients with cleidocranial dysplasia. Hum Mutat 2003: 22: 104.

76. Anthonappa RP, Yan-Hui F, King NM et al. Novel complex disease allele mutations in cleidocranial dysplasia patients. J Oral Pathol Med 2014: 43 $798-800$.

77. Ott CE, Leschik G, Trotier F et al. Deletions of the RUNX2 gene are present in about $10 \%$ of individuals with cleidocranial dysplasia. Hum Mutat 2010: 31: E1587-E1593.

78. Machuca-Tzili L, Monroy-Jaramillo N, Gonzalez-del Angel A et al. New mutations in the CBFA1 gene in two Mexican patients with cleidocranial dysplasia. Clin Genet 2002: 61: 349-353.

79. Chen T, Hou J, Hu LL et al. A novel small deletion mutation in RUNX2 gene in one Chinese family with cleidocranial dysplasia. Int J Clin Exp Pathol 2014: 7: 2490-2495.

80. Wang S, Zhang S, Wang $\mathrm{Y}$ et al. Cleidocranial dysplasia syndrome: clinical characteristics and mutation study of a Chinese family. Int J Clin Exp Med 2013: 6: 900-907.

81. Zhou G, Chen Y, Zhou L et al. CBFA1 mutation analysis and functional correlation with phenotypic variability in cleidocranial dysplasia. Hum Mol Genet 1999: 8: 2311-2316. 


\section{Jaruga et al.}

82. Lee MT, Tsai $\mathrm{AC}, \mathrm{Chou} \mathrm{CH}$ et al. Intragenic microdeletion of RUNX2 is a novel mechanism for cleidocranial dysplasia. Genomic Med 2008: 2: $45-49$.

83. Cohen MM Jr. Perspectives on RUNX genes: an update. Am J Med Genet A 2009: 149A: 2629-2646.

84. Izumi K, Yahagi N, Fujii Y et al. Cleidocranial dysplasia plus vascular anomalies with 6p21.2 microdeletion spanning RUNX2 and VEGF. Am J Med Genet A 2006: 140: 398-401.

85. Hansen L, Riis AK, Silahtaroglu A et al. RUNX2 analysis of Danish cleidocranial dysplasia families. Clin Genet 2011: 79: 254-263.

86. Pal T, Napierala D, Becker TA et al. The presence of germ line mosaicism in cleidocranial dysplasia. Clin Genet 2007: 71: 589-591.

87. Shibata A, Machida J, Yamaguchi S et al. Characterisation of novel RUNX2 mutation with alanine tract expansion from Japanese cleidocranial dysplasia patient. Mutagenesis 2015: 31: 61-67.
88. Wu LZ, Xu XY, Liu YF et al. Expansion of polyalanine tracts in the QA domain may play a critical role in the clavicular development of cleidocranial dysplasia. J Genet 2015: 94: 551-553.

89. Mastushita M, Kitoh H, Subasioglu A et al. A glutamine repeat variant of the RUNX2 gene causes cleidocranial dysplasia. Mol Syndromol 2014: 6: $50-53$.

90. Ryoo HM, Kang HY, Lee SK et al. RUNX2 mutations in cleidocranial dysplasia patients. Oral Dis 2010: 16: 55-60.

91. Camilleri S, McDonald F. Runx2 and dental development. Eur J Oral Sci 2006: 114: 361-373.

92. Lee KE, Seymen F, Ko J et al. RUNX2 mutations in cleidocranial dysplasia. Genet Mol Res 2013: 12: 4567-4574. 